\title{
Longitudinal association between autistic traits and affinity for hikikomori in Japanese university students
}

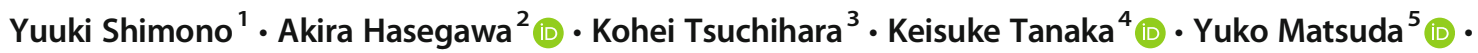 \\ Yoshihiko Kunisato ${ }^{6}$ D
}

Accepted: 11 December 2020 / Published online: 18 January 2021

(C) The Author(s) 2021

\begin{abstract}
The affinity for hikikomori represents the desire to be withdrawn, as well as to entertain an empathetic attitude towards withdrawn individuals. It is composed of two subdimensions, the maladaptive desire for hikikomori, and empathy for others with hikikomori. This longitudinal study examined whether autistic traits predicted the affinity for hikikomori. At the baseline assessment, undergraduate and graduate students in Japan $(N=272)$ completed the Autism-Spectrum Quotient (AQ), the Affinity for Hikikomori Scale in University Students, and measures assessing academic failures and interpersonal conflicts. They also completed all measures excluding the AQ eight weeks later. Structural equation modeling indicated that difficulties in social interaction aspects of autistic traits were positively associated with academic failures at Time 2 even after controlling for academic failures at Time 1 . In addition, difficulties in social interaction were positively related to the desire for hikikomori at Time 2 indirectly via academic failures at Time 2 after controlling for the desire for hikikomori at Time 1. Difficulties in social interaction were also directly associated with the increased desire for hikikomori at Time 2. These findings suggest that autistic traits, and especially difficulties in social interaction, are predictors of the maladaptive aspect of the affinity for hikikomori.
\end{abstract}

Keywords Hikikomori $\cdot$ Social withdrawal $\cdot$ Autistic traits $\cdot$ Stressor $\cdot$ Stress generation

Hikikomori is a Japanese word meaning prolonged social withdrawal. According to the guidelines for hikikomori announced by Japan's Ministry of Health, Labour and Welfare, it is defined as "a situation where a person without psychosis is withdrawn into his/her home for more than six months and does not participate in society such as attending school and/or work" (Saito,

\footnotetext{
${ }^{1}$ Although the definition of hikikomori has been different in different studies, they have been nearly identical. Therefore, we will not focus on these minor differences in definitions.
}

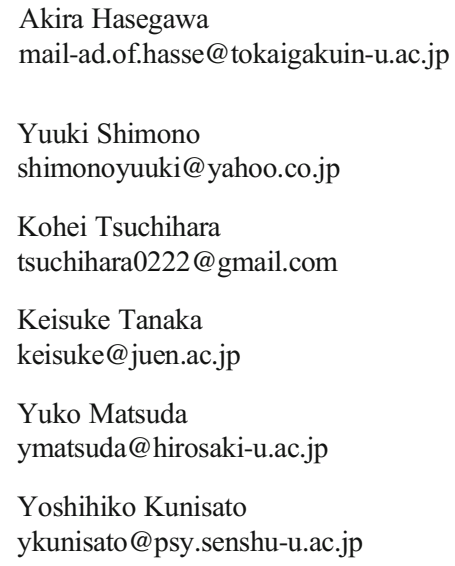

2010; English translation by Kato et al., 2018). ${ }^{1}$ As described in this definition, individuals with hikikomori are outside of education and employment, suggesting that hikikomori might be closely associated with significant functional impairments. However, a community-based survey conducted by Koyama et al. (2010) showed that approximately half the hikikomori cases
1 Center for Clinical Psychology, Tokai Gakuin University, 5-68 Naka-kirino, Kakamigahara City, Gifu 504-8511, Japan

2 Faculty of Human Relations, Tokai Gakuin University, 5-68 Naka-kirino, Kakamigahara City, Gifu 504-8511, Japan

3 subLime design Co., Ltd., 7-5-11-2F, Nishishinjuku, Shinjuku-ku, Tokyo 160-0023, Japan

4 Graduate School of Education, Joetsu University of Education, 1-Yamayashiki-machi, Joetsu-shi, Niigata 943-8512, Japan

5 Graduate Schools of Health Sciences, Hirosaki University, 66-1, Hon-cho, Hirosaki-shi, Aomori 036-8564, Japan

6 Department of Psychology, School of Human Sciences, Senshu University, 2-1-1, Higashimita, Tama-ku, Kawasaki-shi, Kanagawa 214-8580, Japan 
did not have a lifetime experience of any psychiatric disorder listed in DSM-IV (American Psychiatric Association, 1994). Therefore, this functional impairment cannot be fully explained by comorbid psychiatric disorders.

Koyama et al. (2010) also reported that $1.2 \%$ of community residents aged 20-49 in Japan had experienced hikikomori in their lifetime. Although cases of hikikomori were first reported in Japan (Furlong, 2008), this phenomenon has also attracted the attention of researchers and clinicians from other countries in the past decade. Indeed, cases of hikikomori have been reported in several other countries and regions including India, South Korea, the United States (Teo et al., 2015), Oman (Sakamoto et al., 2005), Spain (Malagón-Amor et al., 2015), Italy (Ranieri, 2015), France (Chauliac et al., 2017), and Hong Kong (Wong et al., 2015).

It has been recently suggested that there are Japanese people that did not show prolonged social withdrawal but wanted to be withdrawn and had an empathetic attitude toward individuals with hikikomori. Watanabe et al. (2010) named these desires and attitudes, the affinity for hikikomori, which were assessed by using a scale that was composed of four items including, "I didn't want to go out when an aversive event occurred" and "I can understand why some people stay in their home or rooms and cannot go out." The affinity for hikikomori in non-clinical university students was related to frequent absence from university classes and past experiences of school non-attendance from elementary, junior high, or high school (Shimono \& Hasegawa, 2018; Shimono et al., 2020). In addition, university students with increased affinity for hikikomori show severe depressive symptoms and a lower quality of life (Shimono et al., 2020), similar to individuals with hikikomori (Koyama et al., 2010; Nonaka \& Sakai, 2014). These similarities indicate the possibility that the affinity for hikikomori might be a precursor of hikikomori, although this conclusion should be made cautiously because longitudinal associations between the affinity for hikikomori and hikikomori have not been examined to date.

Specific studies have examined factors that increase the affinity for hikikomori. For example, previous cross-sectional studies of university students have shown that factors such as social anxiety (Arai et al., 2015), frequent exposure to academic failure, increased use of avoidance coping, and decreased use of positive reappraisal coping (Shimono \& Hasegawa, 2018) were associated with increased affinity for hikikomori. However, additional factors are needed to explain the affinity for hikikomori because these variables at most explained only about $15 \%$ of the variance. Furthermore, causality between the affinity for hikikomori and other variables is unclear because all the previous studies had been cross-sectional.

It is possible that autistic traits, which include low social motivation, poor communication, and narrow interests, might increase the affinity for hikikomori. Autistic traits are assessed by self-report measures, such as the Autism-Spectrum
Quotient (AQ; Baron-Cohen et al., 2001). Several different factor models of the AQ have been proposed (Austin, 2005; Hurst et al., 2007; Kloosterman et al., 2011; Lau et al., 2013; Stewart \& Austin, 2009). However, the two-factor model of Hoekstra et al. (2008) has the advantage of being simple and overlapping with the cardinal symptoms of ASD described in DSM-5 (American Psychiatric Association, 2013; see also Horibe \& Hasegawa, 2020). In this model, autistic traits are composed of difficulties in social interaction exemplified by an unwillingness to approach social situations, difficulties in communicating with others, deficits in empathetic abilities, and attention to detail that include a preference for details and patterns. $^{2}$

Research has suggested that autistic traits might be a factor in increasing the affinity for hikikomori. For example, Kuramoto (2008) reported that a group that had experienced periods of hikikomori had a higher total AQ score than a group that had never experienced hikikomori. In addition, Katsuki et al. (2020) demonstrated that clinically hikikomori patients currently exhibiting hikikomori symptoms had a higher total AQ score than patients without current hikikomori. Therefore, if the affinity for hikikomori is a precursor of hikikomori, it is plausible that affinity for hikikomori would be positively associated with autistic traits. Difficulties in social interaction aspects of autistic traits in particular, might directly increase the affinity for hikikomori because university students with autistic traits might tire of interacting with others.

In addition, university students with higher AQ scores have reported lower perceived social support from their friends and families than those with lower AQ scores (Kanai, 2010). Therefore, university students with especially high difficulties in social interaction subscale scores might be prone to frequent academic failures because they fail to obtain sufficient social support. These stresses in the academic domain may increase the affinity for hikikomori, as reported by Shimono and Hasegawa (2018). Furthermore, the attention to detail subscale reflects a narrowing of interests (Hoekstra et al., 2008). Therefore, university students with a higher attention to detail subscale score might reject academic programs that do not interest them. As a result, the attention to detail aspect of autistic traits might also increase the affinity for hikikomori via academic failures.

The current study was designed to examine whether autistic traits increase the affinity for hikikomori in Japanese university students. An eight-week longitudinal study was conducted in the same semester to examine the causality of this relationship. This study, which is based on Shimono and Hasegawa (2018), examined the role of academic stressors (i.e., academic failures) and interpersonal stressors (i.e.,

\footnotetext{
${ }^{2}$ Hoekstra et al. (2008) named the former subscale as social interaction. However, this subscale assesses difficulties in social interaction. Therefore, we termed this subscale, the difficulties in social interaction subscale.
} 
interpersonal conflicts) in mediating between autistic traits and the affinity for hikikomori. We also examined the influences of autistic traits on two subdimensions of the affinity for hikikomori: the desire for hikikomori, and empathy for others with hikikomori, proposed in a recent study (Shimono et al., 2020). The former represents students' desire to withdraw from daily life such as school activities and stay in their homes, whereas the latter represents a gentle and non-critical attitude towards others who are in the state of withdrawal. The desire for hikikomori is conceptualized as a maladaptive aspect of the affinity of hikikomori because it is associated with the absence from university classes, depression, the quality of life, and satisfaction with friend relationships, whereas empathy for others with hikikomori is not associated with these variables (Shimono et al., 2020).

It was expected that autistic traits would be especially predictive of the desire for hikikomori. This study hypothesized that both difficulties in social interaction aspects and attention to detail aspect of autistic traits increase the desire for hikikomori indirectly via the increase in academic failures, whereas difficulties in social interaction directly increase the desire for hikikomori. We also explored the relationships between autistic traits and empathy for others with hikikomori.

\section{Method}

\section{Participants}

Participants were recruited in classes at Hirosaki University, Joetsu University of Education, Senshu University, and Tokai Gakuin University in Japan. They responded to questionnaires in their classless during May of 2018 (Time 1) and again in the same classes eight weeks later (Time 2). We set eight weeks as the interval between Times 1 and 2 because the affinity for hikikomori might change during this interval, although the test-retest correlation of the affinity for hikikomori has not been established. In addition, we could conduct two surveys with an eight-week interval within the same semester.

Undergraduate and graduate students responded to a packet of questionnaires at Time 1 and 2 ( $n s=376$ and 393, respectively). The data of participants that did not participate at two time-points, participants with missing data on any questionnaire at either time, and those returning the questionnaires after the survey day, such that the duration between Times 1 and 2 exceeded eight weeks, were excluded from the analyses. In addition, the data of five participants that were 30 years or older were excluded from the analysis to avoid possible age effects. ${ }^{3}$ The final sample comprised 272 students (109 men, 163 women). The mean age of the final sample was 19.30 ( $S D=1.51$, age range $18-26$ years).

\footnotetext{
${ }^{3}$ Nearly the same results were obtained when age outliers were excluded.
}

\section{Measures}

Autistic Traits The Autism-Spectrum Quotient (AQ; BaronCohen et al., 2001) was used to assess autistic traits. This scale is composed of 50 items, in which each is rated on a 4point rating scale using anchors ranging from 1 (definitely disagree) to 4 (definitely agree). The Japanese version of AQ (Wakabayashi et al., 2004) was used in this study. BaronCohen et al. (2001) proposed a scoring system in which the items are scored as 1 for a response in the autistic direction and 0 for a non-autistic response. However, this study treated the response scale as a four-point rating scale following the scoring method devised by Austin (2005) and Hoekstra et al., (2007), because the method yields more variance than the $0 / 1$ scoring system, which was considered more suitable for detecting associations between autistic traits and other variables. This scale was initially assumed to have a five-factor structure (Baron-Cohen et al., 2001). However, for reasons explained above, the current study adopted the two-factor model proposed by Hoekstra et al. (2008). In this model, autistic traits are composed of difficulties in social interaction and attention to detail. Difficulties in social interaction are a higher-order factor encompassing lower-order dimensions of social skills, attention switching, communication, and imagination, which are the four dimensions proposed by Baron-Cohen et al. (2001). On the other hand, attention to detail in the model of Hoekstra et al. is identical to the subscale proposed by Baron-Cohen et al. (2001). Attention to detail subscale scores that sum the scores of the ten items and difficulties in social interaction subscale scores that sum the scores of the other 40 items were calculated. Cronbach's alpha for difficulties in social interaction was high $(\alpha=.86)$, whereas that for attention to detail was moderate $(\alpha=.65)$.

Affinity for Hikikomori The Affinity for Hikikomori Scale in University Students (Shimono et al., 2020) assessed the two dimensions of the affinity for hikikomori: the desire for hikikomori, and empathy for others with hikikomori. The desire for hikikomori subscale and empathy for others with hikikomori subscale comprises 10 and 6 items, respectively. Sample items of each subscale include, "I didn't want to go out when an aversive event occurred" and "there must be a reason why some individuals cannot go out," respectively. Participants were asked if each item applies to themselves and rated each item on a 4-point rating scale with the anchors ranging from 1 (no) to 4 (yes). We followed the instruction in the original Affinity of Hikikomori Scale (Watanabe et al., 2010) and did not clarify the period that participants were inquired about in the scale. Cronbach's alpha of the desire for hikikomori subscale and empathy for others with hikikomori subscale were .82 and .78 at Time 1, and .84 and .87 at Time 2 , respectively. 
Table 1. Descriptive statistics of each measure.

\begin{tabular}{lccccrr}
\hline & $n$ & $M$ & $S D$ & Range & Skewness & Kurtosis \\
\hline Time 1 & & & & & & \\
Difficulties in social interaction & 260 & 95.30 & 14.53 & $58-145$ & 0.04 & 0.26 \\
Attention to detail & 263 & 21.11 & 4.20 & $9-33$ & 0.20 & 0.18 \\
Desire for hikikomori & 269 & 26.31 & 6.02 & $10-40$ & -0.08 & -0.34 \\
Empathy for others with hikikomori & 264 & 20.11 & 3.18 & $6-24$ & -0.86 & 1.50 \\
Academic failures & 269 & 18.17 & 5.67 & $9-35$ & 0.42 & -0.27 \\
Interpersonal conflicts & 268 & 16.50 & 5.78 & $9-36$ & 0.79 & 0.38 \\
Time 2 & & & & & & -0.11 \\
Desire for hikikomori & 268 & 27.00 & 6.04 & $10-40$ & -0.29 \\
Empathy for others with hikikomori & 271 & 19.87 & 3.72 & $6-24$ & -1.05 & 1.25 \\
Academic failures & 271 & 20.06 & 5.86 & $9-36$ & 0.07 & -0.35 \\
Interpersonal conflicts & 271 & 17.15 & 5.94 & $9-36$ & 0.75 & 0.24 \\
\hline
\end{tabular}

Stressors This study assessed two kinds of stressors that are frequently encountered by Japanese university students: Academic failures and interpersonal conflicts. We used the same items as those used by Shimono and Hasegawa (2018). Items in the academic failures subscale were identical to the established scale or their modifications (e.g., "I had bad exam results," "I did not finish the reports by the due date," and 'I couldn't answer the professors' questions well in class."). Interpersonal conflict items included those in a subscale of the Interpersonal Stress Event Scale (Hashimoto, 1997; e.g., "I quarreled with my acquaintance," "I was insulted by others," and "I disagreed with my acquaintance."). Both subscales were composed of 9 items, and each item was rated on a 4-point rating scale with the anchors ranging from 1 (not at all) to 4 (frequently). At Time 1, the participants were inquired about the frequency of encountering each stressor during the past four weeks, whereas at Time 2, they were inquired about the frequency of encountering each stressor during the past eight weeks. This difference was because Time 1 was approximately one month after the start of a new semester, and the participants responded about experiences of stressors during these four weeks, whereas they responded about stressors during the eight-week follow-up period at Time 2. Cronbach's alpha of academic failures and interpersonal conflicts were .83 and .87 at Time 1 , and .84 and .89 at Time 2 , respectively.

Table 2. Correlations between variables

\begin{tabular}{|c|c|c|c|c|c|c|c|c|c|}
\hline & 1 & 2 & 3 & 4 & 5 & 6 & 7 & 8 & 9 \\
\hline \multicolumn{10}{|l|}{ Time } \\
\hline \multicolumn{10}{|l|}{ 1. Difficulties in social interaction } \\
\hline 2. Attention to detail & $\begin{array}{c}.01 \\
{[-.12, .13]}\end{array}$ & & & & & & & & \\
\hline 3. Desire for hikikomori & $\begin{array}{l}.53 * * * \\
{[.44, .62]}\end{array}$ & $\begin{array}{c}.04 \\
{[-.08, .16]}\end{array}$ & & & & & & & \\
\hline 4. Empathy for others with hikikomori & $\begin{array}{c}.06 \\
{[-.07, .18]}\end{array}$ & $\begin{array}{c}.13 * \\
{[.01, .25]}\end{array}$ & $\begin{array}{c}.34 * * * \\
{[.23, .45]}\end{array}$ & & & & & & \\
\hline 5. Academic failures & $\begin{array}{c}.32 * * * \\
{[.21, .43]}\end{array}$ & $\begin{array}{c}-.09 \\
{[-.21, .04]}\end{array}$ & $\begin{array}{c}.23 * * * \\
{[.11, .34]}\end{array}$ & $\begin{array}{c}-.01 \\
{[-.14, .11]}\end{array}$ & & & & & \\
\hline 6. Interpersonal conflicts & $\begin{array}{c}.14 * \\
{[.02, .26]}\end{array}$ & $\begin{array}{c}.02 \\
{[-.11, .14]}\end{array}$ & $\begin{array}{c}.11 \\
{[-.00, .23]}\end{array}$ & $\begin{array}{c}-.02 \\
{[-.14, .11]}\end{array}$ & $\begin{array}{c}.61 * * * \\
{[.53, .68]}\end{array}$ & & & & \\
\hline Time 2 & & & & & & & & & \\
\hline 7. Desire for hikikomori & $\begin{array}{c}.53 * * * \\
{[.44, .62]}\end{array}$ & $\begin{array}{c}.08 \\
{[-.04, .20]}\end{array}$ & $\begin{array}{c}.80 * * * \\
{[.76, .84]}\end{array}$ & $\begin{array}{c}.28 * * * \\
{[.17, .39]}\end{array}$ & $\begin{array}{c}.23 * * * \\
{[.11, .34]}\end{array}$ & $\begin{array}{c}.09 \\
{[-.03, .21]}\end{array}$ & & & \\
\hline 8. Empathy for others with hikikomori & $\begin{array}{c}.07 \\
{[-.06, .20]}\end{array}$ & $\begin{array}{c}.18^{* *} \\
{[.06, .29]}\end{array}$ & $\begin{array}{c}.38 * * * \\
{[.28, .49]}\end{array}$ & $\begin{array}{c}.54 * * * * \\
{[.45, .63]}\end{array}$ & $\begin{array}{c}.01 \\
{[-.11, .13]}\end{array}$ & $\begin{array}{c}-.11 \\
{[-.23, .01]}\end{array}$ & $\begin{array}{c}.42 * * * \\
{[.32, .52]}\end{array}$ & & \\
\hline 9. Academic failures & $\begin{array}{c}.33 * * * \\
{[.22, .44]}\end{array}$ & $\begin{array}{c}.01 \\
{[-.11, .13]}\end{array}$ & $\begin{array}{c}.30 * * * \\
{[.19, .41]}\end{array}$ & $\begin{array}{c}.13 * \\
{[.01, .25]}\end{array}$ & $\begin{array}{c}.53 * * * \\
{[.44, .62]}\end{array}$ & $\begin{array}{c}.30 * * * \\
{[.19, .41]}\end{array}$ & $\begin{array}{c}.40 * * * \\
{[.30, .50]}\end{array}$ & $\begin{array}{c}.16^{* *} \\
{[.04, .28]}\end{array}$ & \\
\hline 10. Interpersonal conflicts & $\begin{array}{c}.12 \\
{[-.00, .25]}\end{array}$ & $\begin{array}{c}.05 \\
{[-.07, .18]}\end{array}$ & $\begin{array}{c}.10 \\
{[-.02, .22]}\end{array}$ & $\begin{array}{c}-.01 \\
{[-.13, .12]}\end{array}$ & $\begin{array}{c}.33 * * * \\
{[.22, .44]}\end{array}$ & $\begin{array}{c}.48 * * * \\
{[.39, .57]}\end{array}$ & $\begin{array}{c}.22 * * * * \\
{[.10, .33]}\end{array}$ & $\begin{array}{c}.01 \\
{[-.11, .13]}\end{array}$ & $\begin{array}{c}.59 * * * \\
{[.51, .67]}\end{array}$ \\
\hline
\end{tabular}

Note: Numbers in parentheses indicate $95 \%$ confidence intervals. $* p<.05, * * p<.01$, *** $p<.001$. 


\section{Procedure}

Students were given a description of the study prior to participation. Only the students that agreed to take part in the study responded to the questionnaires. Participants completed all three scales at Time 1 . They also completed the same scales, excluding the AQ, at Time 2. Participants wrote their birthdays and the last four digits of their mobile phone numbers on spaces provided in the questionnaire packet, which was used to match the data from the two surveys. The Ethics Committee of Tokai Gakuin University approved this study.

\section{Statistical Analysis}

Data were analyzed by allowing for missing data except in the case of paired $t$-tests. Descriptive statistics and $t$-test were conducted using SPSS ver. 23 (IBM Corporation), and all other analyses were conducted using Mplus 8.3 (Muthén \& Muthén, 1998-2017). A paired $t$-test was conducted to examine for any statistically significant differences in the two dimensions of the affinity for hikikomori and the two types of stressors at two timepoints. We calculated simple correlations among all variables. Structural equation modeling (SEM) was conducted to examine the assumed model. Missing data when conducting correlation analysis and SEM were treated by using the Full Information Maximum Likelihood (FIML) method.

\section{Results}

Descriptive statics were shown in Table 1. A paired $t$-test was performed using the data from participants that completed each subscale at both Time 1 and 2 without missing values. The desire for hikikomori subscale scores were significantly higher at Time 2 than Time $1(t(264)=2.60, p=.010, d=$ $0.10)$. However, there was no significant difference between empathy for others with hikikomori at Time 1 and $2(t(262)=$ $-1.00, p=.320, d=-0.06)$. In addition, participants had experienced academic failures more frequently at Time 2 than Time $1(t(267)=5.28, p<.001, d=0.31)$, while they experienced a similar amount of interpersonal conflicts at Time 1 and $2(t(266)=1.60, p=.110, d=0.10)$. Although the desire for hikikomori and academic failures significantly increased from Time 1 to Time 2, the effect sizes were small.

Table 2 shows the correlations between each variable. It can be seen that the test-retest correlation of the desire for hikikomori is strong $(r=.80)$, whereas that of empathy for others with hikikomori is moderate $(r=.54)$.

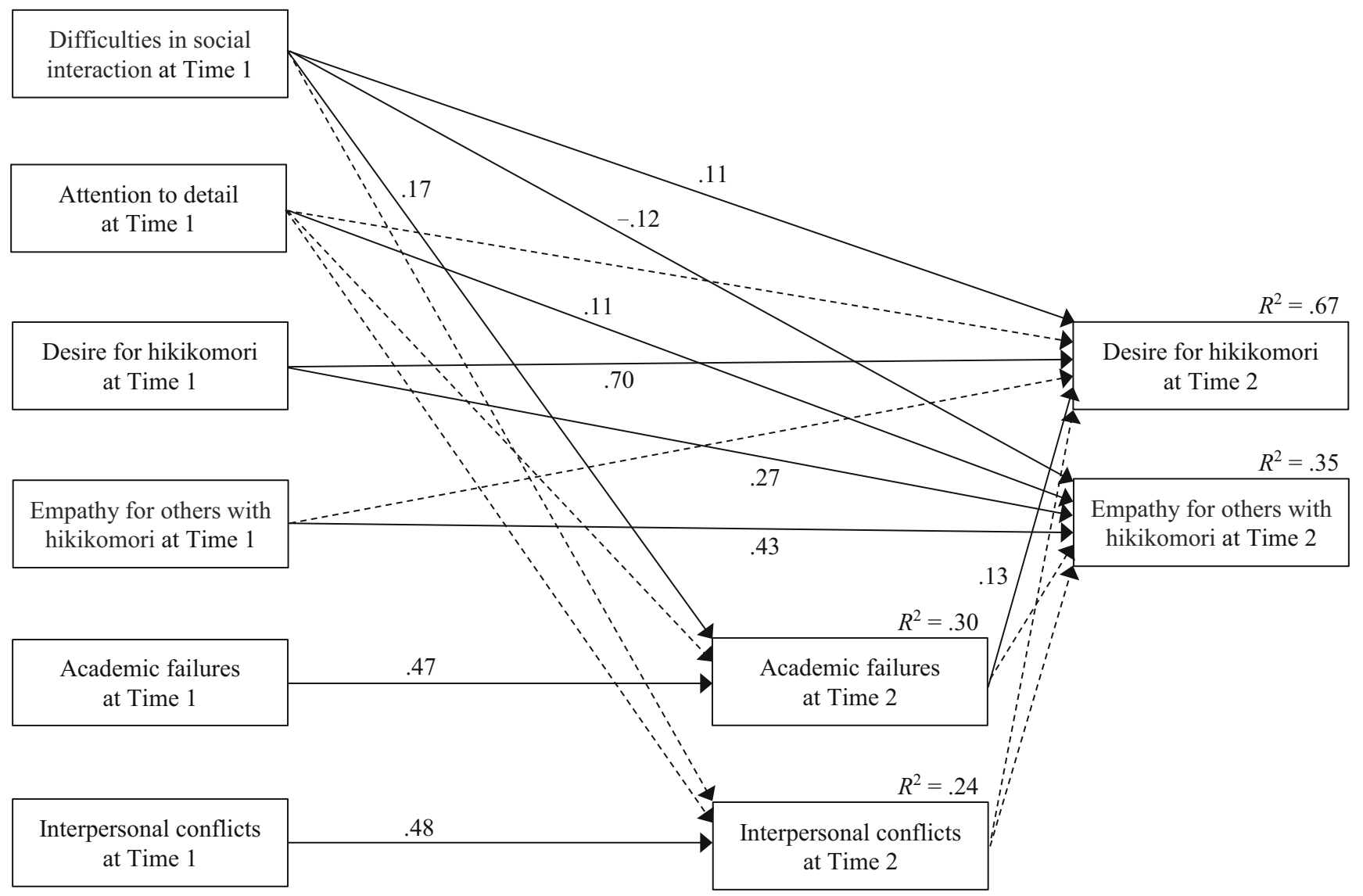

Fig. 1 Path model examining the assumed model $(N=272)$. All standardized coefficients and $R^{2}$ shown here are significant $(p<.05)$, and non-significant paths are shown in dashed lines. Error variables and covariances are omitted 
SEM with FIML was conducted to examine whether the two subdimensions of autistic traits at Time 1 were related to the desire for hikikomori and empathy for others with hikikomori at Time 2 directly and indirectly via academic failures and interpersonal conflicts at Time 2. Because the desire for hikikomori at Time 1 was positively correlated with empathy for others with hikikomori at Time 2 and vice versa, it was possible that the two dimensions of the affinity for hikikomori might be a mutually enhancing relationship. Since this relationship was theoretically justified (e.g., those who have a desire for hikikomori can have a gentle and noncritical attitude towards others who are in a state of withdrawal), we also examined whether the desire for hikikomori at Time 1 was associated with an increase in empathy for others with hikikomori at Time 2 and vice versa. The model examined whether other variables predicted academic failures, interpersonal conflicts, the desire for hikikomori, and empathy for others with hikikomori at Time 2 after controlling for the influences of the same variables at Time 1. Correlations among each variable at Time 1 , those of two kinds of stressors at Time 2, and those of two dimensions of the affinity for hikikomori at Time 2 were allowed. Figure 1 shows the results. The model provided an acceptable fit to the data $\left(\chi^{2}\right.$ $(10)=26.51, p=.004 ; \mathrm{CFI}=.98 ; \mathrm{RMSEA}=.08)$.

Difficulties in social interaction were associated with an increase in academic failures at Time 2 , and both variables were associated with an increase in the desire for hikikomori at Time 2. On the other hand, attention to detail was not significantly related to academic failures or the desire for hikikomori at Time 2. Attention to detail and the desire for hikikomori at Time 1 were associated with an increase in empathy for others with hikikomori at Time 2, whereas difficulties in social interaction were associated with a decrease in empathy for others with hikikomori. A bias-corrected bootstrap test with 10,000 bootstrap re-samples was conducted to determine the significance of indirect effects. The result indicated that difficulties in social interaction were positively associated with the desire for hikikomori at Time 2 , indirectly via academic failures at Time $2(\beta=.022$, 95\% CI: $[.004, .054])$.

\section{Discussion}

This eight-week longitudinal study conducted at two timepoints examined whether autistic traits were directly related to the two subdimensions of the affinity for hikikomori, or if they were indirectly related via increased exposure to stressors. The results indicated that difficulties in social interaction aspects of autistic traits were positively associated with academic failures at Time 2 , even after controlling for academic failures at Time 1. A previous study has reported that university students with higher autistic traits had lower perceived social support from friends and families (Kanai, 2010).
It is possible that difficulties in social interaction aspects of autistic traits decrease social support from friends, and in turn, increase academic failures. In contrast, attention to detail aspect of autistic traits was not concurrently or prospectively associated with academic failures, suggesting that attention to detail does not reduce academic performance. Furthermore, the results indicated a non-significant association between difficulties in social interaction at the baseline and interpersonal conflicts at Time 2, which is consistent with a previous finding of a very weak concurrent association between these variables that were non-significant after controlling for attention-deficit/hyperactivity symptoms (Horibe \& Hasegawa, 2020). It is suggested that difficulties in social interaction aspects of autistic traits were a factor leading to academic stressors but not interpersonal stressors.

The present study also demonstrated that academic failures at Time 2 were positively associated with the desire for hikikomori at Time 2 after controlling for the desire for hikikomori at the baseline. The finding that the desire for hikikomori was positively associated with academic failures but not interpersonal conflicts is consistent with a similar association reported by Shimono and Hasegawa (2018) by using the original four items of the Affinity for Hikikomori Scale. Students that experience frequent academic failures might become disgusted or anxious about university classes, and increase the desire for hikikomori. On the other hand, students that experience interpersonal conflicts might reduce the frequency of facing others they had quarreled, thereby preventing any increase of the desire for hikikomori.

The hypothesis of this study was supported by the mediational analysis, which indicated that academic failures mediated the association between difficulties in social interaction and the desire for hikikomori. Moreover, difficulties in social interaction had a direct positive association with the increase in the desire for hikikomori eight weeks later, which was consistent with our hypothesis. This might be because students having more difficulties in social interaction are unwilling to approach social situations, become tired of interacting with others and increase the desire for hikikomori.

The result of SEM showed that difficulties in social interaction at Time 1 were negatively related to empathy for others with hikikomori at Time 2, although it should be noted that the simple correlation between these variables was not significant. Students having more difficulties in social interaction were less likely to show empathetic attitudes towards others and, therefore, might develop a critical attitude towards others with hikikomori. The findings that difficulties in social interaction were associated with an increase in the desire for hikikomori, and a decrease in the empathy for others with hikikomori were consistent with the suggestion by Shimono et al. (2020) that the two subdimensions of the affinity for hikikomori are distinct constructs.

Furthermore, attention to detail at Time 1 was positively associated with an increase in empathy for others with hikikomori at Time 2 . It is possible that students that prefer 
details and patterns consider themselves as being similar to individuals with hikikomori because these individuals with a narrow range of interest may prefer hobbies such as computing alone at home, although this interpretation is speculative and need further investigation.

We also found that the desire for hikikomori at the baseline was positively related to empathy for others with hikikomori eight weeks later. This could be because students desiring to withdraw, develop a gentle, non-critical attitude towards others with hikikomori. On the other hand, empathy for others with hikikomori did not predict the desire for hikikomori.

This study demonstrated that difficulties in social interaction aspects of autistic traits were positively related to the desire for hikikomori directly or indirectly via increased academic failures. These findings contribute to specifying factors that would increase the affinity for hikikomori. In addition, although this was not the main focus of this study, our findings could contribute to advancing studies on the stress generation because the findings suggested that difficulties in social interaction are a factor to generate academic stressors. While studies on the stress generation have indicated specific factors associated with increased stressors, including childhood maltreatment, negative inferential styles, and excessive reassurance-seeking (Liu, 2013, for review), the present findings suggest that autistic traits are an additional factor to generate stressors. More research is necessary to examine this possibility in detail.

This study had some limitations. Firstly, the participants of this study were all undergraduate and graduate students. Therefore, future studies are needed to replicate our findings with populations other than university students. Secondly, this study wholly relied on self-report measures for assessing each variable, which is similar to previous studies that have assessed the affinity for hikikomori. However, future work should use interview-based or behavioral measures to examine the predictors of the affinity for hikikomori. Finally, the associations between stressors and the affinity for hikikomori were examined concurrently at Time 2 . Therefore, it is suggested that a three-wave survey investigating the indirect effects from autistic traits to stressors, and from stressors to the affinity for hikikomori, should be undertaken in the future.

Funding The authors received no financial support for the research, authorship, and publication of this article.

Data Availability The datasets generated during and/or analyzed during the current study are available from the corresponding author on reasonable request.

\section{Compliance with Ethical Standards}

Ethical Approval All procedures performed in studies involving human participants were in accordance with the ethical standards of the institutional research committee and with the 1964 Helsinki declaration and its later amendments or comparable ethical standards.
Informed Consent Informed consent was obtained from all individual participants included in the study.

Conflict of Interest The authors declared that they had no conflicts of interest with respect to their authorship or the publication of this article.

Open Access This article is licensed under a Creative Commons Attribution 4.0 International License, which permits use, sharing, adaptation, distribution and reproduction in any medium or format, as long as you give appropriate credit to the original author(s) and the source, provide a link to the Creative Commons licence, and indicate if changes were made. The images or other third party material in this article are included in the article's Creative Commons licence, unless indicated otherwise in a credit line to the material. If material is not included in the article's Creative Commons licence and your intended use is not permitted by statutory regulation or exceeds the permitted use, you will need to obtain permission directly from the copyright holder. To view a copy of this licence, visit http://creativecommons.org/licenses/by/4.0/.

\section{References}

American Psychiatric Association. (1994). Diagnostic and statistical manual of mental disorders (4th ed.).

American Psychiatric Association. (2013). Diagnostic and statistical manual of mental disorders (5th ed.). https://doi.org/10.1176/appi. books. 9780890425596.

Arai, H., Hironaka, Y., \& Kondo, K. (2015). Syakou-huan shyoujou to taijinn-teki jiko-kouryokukan ga daigakusei no hikikomorishinwasei ni ataeru eikyou [the effects of social anxiety symptoms and social self-efficacy on affinity for social withdrawal in university students]. Japanese Journal of Personality, 24(1), 1-14. https:// doi.org/10.2132/personality.24.1.

Austin, E. J. (2005). Personality correlates of the broader autism phenotype as assessed by the Autism Spectrum Quotient (AQ). Personality and Individual Differences, 38(2), 451-460. https:// doi.org/10.1016/j.paid.2004.04.022.

Baron-Cohen, S., Wheelwright, S., Skinner, R., Martin, J., \& Clubley, E. (2001). The Autism-Spectrum Quotient (AQ): Evidence from Asperger syndrome/high-functioning autism, males and females, scientists and mathematicians. Journal of Autism and Developmental Disorders, 31(1), 5-17. https://doi.org/10.1023/A: 1005653411471.

Chauliac, N., Couillet, A., Faivre, S., Brochard, N., \& Terra, J. L. (2017). Characteristics of socially withdrawn youth in France: A retrospective study. International Journal of Social Psychiatry, 63(4), 339344. https://doi.org/10.1177/0020764017704474.

Furlong, A. (2008). The Japanese hikikomori phenomenon: Acute social withdrawal among young people. The Sociological Review, 56(2), 309-325. https://doi.org/10.1111/j.1467-954X.2008.00790.x.

Hashimoto, T. (1997). Daigakusei ni okeru taijin stress event bunrui no kokoromi [categorization of interpersonal stress events among undergraduates]. Japanese Journal of Social Psychology, 13(1), 64 75. https://doi.org/10.14966/jssp.KJ00003724759.

Hoekstra, R. A., Bartels, M., Verweij, C. J. H., \& Boomsma, D. I. (2007). Heritability of autistic traits in the general population. Archives of Pediatrics \& Adolescent Medicine, 161(4), 372-377. https://doi.org/ 10.1001/archpedi.161.4.372.

Hoekstra, R. A., Bartels, M., Cath, D. C., \& Boomsma, D. I. (2008). Factor structure, reliability and criterion validity of the AutismSpectrum Quotient (AQ): A study in Dutch population and patient groups. Journal of Autism and Developmental Disorders, 38(8), 1555-1566. https://doi.org/10.1007/s10803-008-0538-x.

Horibe, K., \& Hasegawa, A. (2020). How autistic traits, inattention and hyperactivity-impulsivity symptoms influence depression in 
nonclinical university students? Mediating role of depressive rumination. Current Psychology, 39, 1543-1551. https://doi.org/10. 1007/s12144-018-9853-3.

Hurst, R. M., Mitchell, J. T., Kimbrel, N. A., Kwapil, T. K., \& NelsonGray, R. O. (2007). Examination of the reliability and factor structure of the Autism Spectrum Quotient (AQ) in a non-clinical sample. Personality and Individual Differences, 43(7), 1938-1949. https:// doi.org/10.1016/j.paid.2007.06.012.

Kanai, Y. (2010). Jihei-shou spectrum keikou wo shimesu daigakusei no yokuutsu ni social support to higai-nenryo ga oyobosu eikyou [effects of social support and paranoia on depression in undergraduate students with symptoms of Autism-Spectrum Disorder]. Japanese Journal of Counseling Science, 43(2), 114-119. https://doi.org/10. 11544/cou.43.2 114.

Kato, T. A., Kanba, S., \& Teo, A. R. (2018). Hikikomori: Experience in Japan and international relevance. World Psychiatry, 17(1), 105106. https://doi.org/10.1002/wps.20497.

Katsuki, R., Tateno, M., Kubo, H., Kurahara, K., Hayakawa, K., Kuwano, N., Kanba, S., \& Kato, T. A. (2020). Autism spectrum conditions in hikikomori: A pilot case-control study. Psychiatry and Clinical Neurosciences, 74(12), 652-658. https://doi.org/10. 1111/pcn.13154.

Kloosterman, P. H., Keefer, K. V., Kelley, E. A., Summerfeldt, L. J., \& Parker, J. D. A. (2011). Evaluation of the factor structure of the Autism-Spectrum Quotient. Personality and Individual Differences, 50(2), 310-314. https://doi.org/10.1016/j.paid.2010. 10.015

Koyama, A., Miyake, Y., Kawakami, N., Tsuchiya, M., Tachimori, H., Takeshima, T., \& The World Mental Health Japan Survey Group, 2002-2006. (2010). Lifetime prevalence, psychiatric comorbidity and demographic correlates of "hikikomori" in a community population in Japan. Psychiatry Research, 176(1), 69-74. https://doi.org/ 10.1016/j.psychres.2008.10.019.

Kuramoto, N. (2008). Syakai-teki hikikomori ni kanyo suru shinri-teki tokusei no kentou [A study of psychological traits of Hikikomori]. Journal of Japanese Clinical Psychology, 26(3), 314-324.

Lau, W. Y. P., Kelly, A. B., \& Peterson, C. C. (2013). Further evidence on the factorial structure of the Autism Spectrum Quotient (AQ) for adults with and without a clinical diagnosis of autism. Journal of Autism and Developmental Disorders, 43(12), 2807-2815. https:// doi.org/10.1007/s10803-013-1827-6.

Liu, R. T. (2013). Stress generation: Future directions and clinical implications. Clinical Psychology Review, 33(3), 406-416. https://doi. org/10.1016/j.cpr.2013.01.005.

Malagón-Amor, Á., Córcoles-Martínez, D., Martín-López, L. M., \& Pérez-Solà, V. (2015). Hikikomori in Spain: A descriptive study. International Journal of Social Psychiatry, 61(5), 475-483. https://doi.org/10.1177/0020764014553003.

Muthén, L. K., \& Muthén, B. O. (1998-2017). Mplus User's Guide (Eighth ed.). Los Angeles, CA: Muthén \& Muthén.

Nonaka, S., \& Sakai, M. (2014). Hikikomori-joutai ga Quality of life ni oyobosu eikyou [the effect of hikikomori on quality of life]. Japanese Journal of Psychology, 85(3), 313-318. https://doi.org/ 10.4992/jjpsy.85.13315.

Ranieri, F. (2015). When social withdrawal in adolescence becomes extreme: The "hikikomori" phenomenon in Italy. Psychiatria $i$
Psychologia Kliniczna, 15(3), 148-151. https://doi.org/10.15557/ PiPK.2015.0023.

Saito, K. (2010). Hikikomori no hyouka: Shien ni kansuru gaidorain [guidelines for assessment and support of hikikomori]. Tokyo, Japan: Japan's Ministry of Health, Labour and Welfare https:// www.mhlw.go.jp/file/06-Seisakujouhou-12000000Shakaiengokyoku-Shakai/0000147789.pdf.

Sakamoto, N., Martin, R. G., Kumano, H., Kuboki, T., \& Al-Adawi, S. (2005). Hikikomori, is it a culture-reactive or culture-bound syndrome? Nidotherapy and a clinical vignette from Oman. International Journal of Psychiatry in Medicine, 35(2), 191-198. https://doi.org/10.2190/7WEQ-216D-TVNH-PQJ1.

Shimono, Y., \& Hasegawa, A. (2018). Daigakusei no gakugyou ni okeru stress taisyo ga hikikomori-shinwasei ni oyobosu eikyou: Coping houryaku to enjo-yousei koudou wo toriagete [the impact for coping strategies and help-seeking behaviors on the affinity for "hikikomori" among Japanese university students]. Japanese Journal of Cognitive Therapy, 11(1), 80-91.

Shimono, Y., Hasegawa, A., Tsuchihara, K., \& Kunisato, Y. (2020). Daigakusei-you hikikomori-shinwasei syakudo no sakusei [development of the affinity for hikikomori scale in university students]. Japanese Journal of Research on Emotions, 27(2), 51-60. https:// doi.org/10.4092/jsre.27.2 51.

Stewart, M. E., \& Austin, E. J. (2009). The structure of the AutismSpectrum Quotient (AQ): Evidence from a student sample in Scotland. Personality and Individual Differences, 47(3), 224-228. https://doi.org/10.1016/j.paid.2009.03.004.

Teo, A. R., Fetters, M. D., Stufflebam, K., Tateno, M., Balhara, Y., Choi, T. Y., Kanba, S., Mathews, C. A., \& Kato, T. A. (2015). Identification of the hikikomori syndrome of social withdrawal: Psychosocial features and treatment preferences in four countries. International Journal of Social Psychiatry, 61(1), 64-72. https://doi. org/10.1177/0020764014535758.

Wakabayashi, A., Tojo, Y., Baron-Cohen, S., \& Wheelwright, S. (2004). Jihei-shou spectrum shisuu (AQ) nihongo-ban no hyoujunka: Koukinou rinsyou-gun to kenjou seijin ni yoru kentou [the AutismSpectrum Quotient (AQ) Japanese version: Evidence from highfunctioning clinical group and normal adults]. Japanese Journal of Psychology, 75(1), 78-84. https://doi.org/10.4992/jjpsy.75.78.

Watanabe, A., Matsui, Y., \& Takatsuka, Y. (2010). Hikikomori oyobi hikikomori-shinwasei wo kitei suru youin no kentou [an examination of the determinants of social withdrawal and affinity for social withdrawal]. Japanese Journal of Psychology, 81(5), 478-484. https:// doi.org/10.4992/jjpsy.81.478.

Wong, P. W. C., Li, T. M. H., Chan, M., Law, Y. W., Chau, M., Cheng, C., Fu, K. W., Bacon-Shone, J., \& Yip, P. S. F. (2015). The prevalence and correlates of severe social withdrawal (hikikomori) in Hong Kong: A cross-sectional telephone-based survey study. International Journal of Social Psychiatry, 61(4), 330-342. https://doi.org/10.1177/0020764014543711.

Publisher's Note Springer Nature remains neutral with regard to jurisdictional claims in published maps and institutional affiliations. 